HIFAN 1723

\title{
New Developments in the Simulation of Advanced Accelerator Concepts
}

\begin{abstract}
By
David L. Bruhwiler, John R. Cary, Benjamin M. Cowan, Kevin Paul, Cameron G. R. Geddes, Paul J. Mullowney, Peter Messmer, Eric Esarey, Estelle Cormier-Michel, Wim Leemans and Jean-Luc Vay
\end{abstract}

Tech-X Corporation

LOASIS Program LBNL

Accelerator Fusion Research Division

Ernest Orlando Lawrence Berkeley National Laboratory

University of California

Berkeley, California 94720

\section{August 2008}

This work was supported by the Director, Office of Science, Office of Fusion Energy Sciences, of the U.S. Department of Energy under Contract No. DE-AC0205CH11231. 


\title{
New Developments in the Simulation of Advanced Accelerator Concepts
}

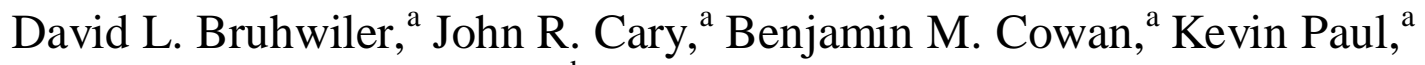 \\ Cameron G. R. Geddes, ${ }^{\mathrm{b}}$ Paul J. Mullowney, ${ }^{\mathrm{a}}$ Peter Messmer, ${ }^{\mathrm{a}}$ \\ Eric Esarey, ${ }^{\mathrm{b}}$ Estelle Cormier-Michel, ${ }^{\mathrm{b}}$ Wim Leemans ${ }^{\mathrm{b}}$ and Jean-Luc Vay ${ }^{\mathrm{b}}$ \\ ${ }^{a}$ Tech-X Corporation, Boulder, CO 80303, USA \\ ${ }^{b}$ LOASIS program, Lawrence Berkeley National Laboratory, Berkeley, CA 94720, USA
}

\begin{abstract}
Improved computational methods are essential to the diverse and rapidly developing field of advanced accelerator concepts. We present an overview of some computational algorithms for laser-plasma concepts and high-brightness photocathode electron sources. In particular, we discuss algorithms for reduced laser-plasma models that can be orders of magnitude faster than their higher-fidelity counterparts, as well as important on-going efforts to include relevant additional physics that has been previously neglected. As an example of the former, we present 2D laser wakefield accelerator simulations in an optimal Lorentz frame, demonstrating $>10 \mathrm{GeV}$ energy gain of externally injected electrons over a $2 \mathrm{~m}$ interaction length, showing good agreement with predictions from scaled simulations and theory, with a speedup factor of 2,000 as compared to standard particle-in-cell.
\end{abstract}

Keywords: laser plasma acceleration, photocathode electron source, particle-in-cell

PACS: 41.75.Ht, 41.75.Jv, 42.70.Qs, 47.11.Bc, 47.11.Fg, 52.38.Kd, 52.65.Rr, 85.60.Ha

\section{INTRODUCTION}

We present an overview of new developments in the simulation of advanced accelerator concepts (AAC). Due to space limitations, we restrict our attention primarily to laser wakefield accelerators (LWFA) and high-brightness electron guns. We further restrict our attention primarily to work in the USA and apologize in advance for any relevant work that we have neglected to mention. New 2D VORPAL [8] simulations in a Lorentz boosted frame [49-52] are presented, demonstrating $>10$ $\mathrm{GeV}$ energy gain of externally injected electrons over a $2 \mathrm{~m}$ interaction length, showing good agreement with predictions from scaled simulations and theory [43], with a speedup factor of $\sim 2,000$ as compared to standard particle-in-cell (PIC).

\section{LASER WAKEFIELD ACCELERATOR (LWFA) SIMULATIONS}

The maximum achievable accelerating gradient is orders of magnitude larger when sustained by the collective fields of a plasma, rather than an evacuated metal structure. This has been demonstrated up to $1 \mathrm{GeV}$ for electrons in laser wakefield accelerator (LWFA) experiments [30]. Plasma-based electron acceleration concepts (see [31] for a review) can sustain longitudinal electric fields on the order of the nonrelativistic 
wave breaking field, $\mathrm{E}_{0}=\mathrm{cm}_{\mathrm{e}} \omega_{\mathrm{p}} / \mathrm{e}$, where $\omega_{\mathrm{p}}=\left(4 \pi \mathrm{n}_{\mathrm{e}} \mathrm{e}^{2} / \mathrm{m}_{\mathrm{e}}\right)^{1 / 2}$ is the plasma frequency at an electron density $n_{\mathrm{e}}$. For $n_{\mathrm{e}}=10^{18} \mathrm{~cm}^{-3}$, the electric field is $E_{0} \sim 100 \mathrm{GV} / \mathrm{m}$, with a phase velocity close to the speed of light.

\section{Explicit Electromagnetic PIC Simulations of LWFA Concepts}

There are many time-explicit electromagnetic particle-in-cell (PIC) codes in use for simulating LWFA experiments, but here we consider only OSIRIS [32-35] and the parallel VORPAL framework [8,36-38], which have recently been benchmarked [39,51]. Given sufficient computing power, electromagnetic PIC codes can simulate the plasma electrons (and ions, if necessary), the laser pulse driving the plasma wake, and the dynamics of electrons that are trapped in the accelerating potential.

Explicit PIC codes must resolve the laser wavelength, which requires small cell sizes and, hence, small time steps, resulting in large simulation grids with long run times. Assuming the number of particles per cell is kept constant, doubling the resolution along each of the three axes (which in turn requires the time step to be halved) will increase the run time by a factor of 16. The standard PIC algorithm is $2^{\text {nd }}$-order accurate [40], so in principle doubling the resolution will reduce errors by a factor of four; however, in practice particle noise can reduce the order of convergence.

Particle noise can be reduced by smoothing the currents on the grid, before coupling them into Maxwell's equations, or by using spline-based particle shapes for the current deposition algorithm. The smoother force interpolation obtained with the same spline-based shape can also be important [41,42]. For long simulation times, it's important to use absorbing boundary conditions, such as perfectly matched layers (PML), to prevent outgoing electromagnetic waves from bouncing back into the domain.

\section{Explicit PIC Simulations with Scaled Parameters}

The maximum energy attained by an electron (or positron) bunch injected into the plasma wake of a laser pulse in the "quasilinear" regime, scales inversely with density [43], while the interaction length increases. Inverse scaling of beam energy with plasma density is also seen in the nonlinear regime [54]. For fixed laser wavelength, the number of grid cells per plasma wavelength increases as the density is reduced. Hence, using half the electron density to double the electron energy means the simulation run-time will increase by more than a factor of eight. As one approaches $\sim 1 \mathrm{~m}$ scale lengths for a $10 \mathrm{GeV}$ LWFA stage, this quickly becomes untenable. It has been shown for quasilinear $\left(a_{0} \sim 1\right)$ stages, that one can dramatically reduce the run time, and retain most of the important physics, including beam loading, by correctly scaling the physical parameters of the problem [43]. Detailed scaling analysis has also been developed for the nonlinear regime [55,57].

\section{Quasistatic and Ponderomotive Guiding Center PIC Simulations}

An alternative to scaling the physical parameters is to use a reduced PIC algorithm that doesn't resolve the laser wavelength or period. One such example is the 
ponderomotive guiding center (PGC) or "envelope" treatment of the laser pulse [44], which has been implemented in VORPAL [45,46] and other codes. A stronger approximation is the quasistatic algorithm, which has been implemented in WAKE (2D cylindrical) and also in the 3D QuickPIC code [47]. QuickPIC has been benchmarked with OSIRIS and VORPAL [39,51].

The PGC algorithm is constrained by the Courant stability criterion, but the laser wavelength need not be resolved, so the longitudinal cell size and, hence, the time step can be much larger than for explicit PIC, resulting in orders of magnitude speedup. The quasistatic algorithm step size can be as large as a fraction of a Rayleigh range, resulting in even greater speedup, but it requires special measures to correctly model the trapping and acceleration of electrons. The PGC algorithm in VORPAL includes particle trapping, making it well suited for simulations of downramp injection [46].

As the laser pulse propagates significant distances through the plasma, its energy depletes, resulting in a broadening of its frequency spectrum (primarily red shifting). The PGC and quasistatic algorithms both fail due to this spectral broadening, before the pulse has completely depleted.

\section{Explicit PIC Simulations in an Optimal Lorentz Frame}

A third possibility for dramatically speeding up laser-plasma simulations, making them practical in $3 \mathrm{D}$ for interaction lengths $>1 \mathrm{~cm}$ and electron energies $>1 \mathrm{GeV}$, is to operate in a boosted Lorentz frame, moving in the direction of the laser pulse. This idea was explored over 10 years ago [48], but didn't work because backward going radiation is upshifted in frequency (and, hence, unresolved) and also amplified, resulting in strong noise that can drive a numerical instability in the plasma. The idea of working in an optimal Lorentz frame was recently rediscovered $[49,50]$ and is being pursued with OSIRIS, VORPAL and Warp [51,52,53] and with other codes.

To date, LWFA boosted-frame VORPAL simulations choose the relativistic factor $\gamma_{\mathrm{b}}$ such that the left-going Lorentz contracted plasma channel is twice the length of the stretched right-going laser pulse, and both remain within the original domain, meaning no moving window algorithm is required. The laser wavelength is still fully resolved and transverse coordinates are unchanged, so the size of the grid remains essentially the same as for a standard lab frame simulation. Hence, the speedup comes from the reduction in the number of time steps: the time step (like the laser wavelength) increases by $\gamma_{\mathrm{b}}\left(1+\beta_{\mathrm{b}}\right)$ and the interaction time (like the plasma length) decreases by $\gamma_{\mathrm{b}}$, so the run time (like the number of time steps) is reduced by the factor $\left(1+\beta_{b}\right) \gamma_{b}{ }^{2} \approx 2 \gamma_{b}{ }^{2}$.

Results of a 2D boosted-frame VORPAL simulation are shown in Fig.'s 1 and 2 below. We consider the very challenging case of a $10 \mathrm{GeV}$ quasilinear stage for an externally injected electron bunch, including a $2 \mathrm{~m}$ plasma density channel with central electron density $\mathrm{n}_{\mathrm{e}}=6 \times 10^{16} \mathrm{~cm}^{-3}$ and a laser pulse with $\lambda=0.8 \mu \mathrm{m}$ and the following dimensionless parameters: $a_{0}=1, k_{p} w_{0}=3.8$ and $k_{p} L=2$ (see e.g. Ref. [43] for definitions). These are similar to the parameters for a $10 \mathrm{GeV}$ stage, as predicted by scaling theory [43]. Simulating this case in $1 \mathrm{D}$ with explicit PIC requires $\sim 10^{8}$ time steps and $\sim 2 \times 10^{4}$ processor hours [51], while a $2 \mathrm{D}$ simulation requires $\sim 500 \mathrm{x}$ more computer time $\left(10^{6}\right.$ processor hours), and a $3 \mathrm{D}$ simulation is essentially impossible. 


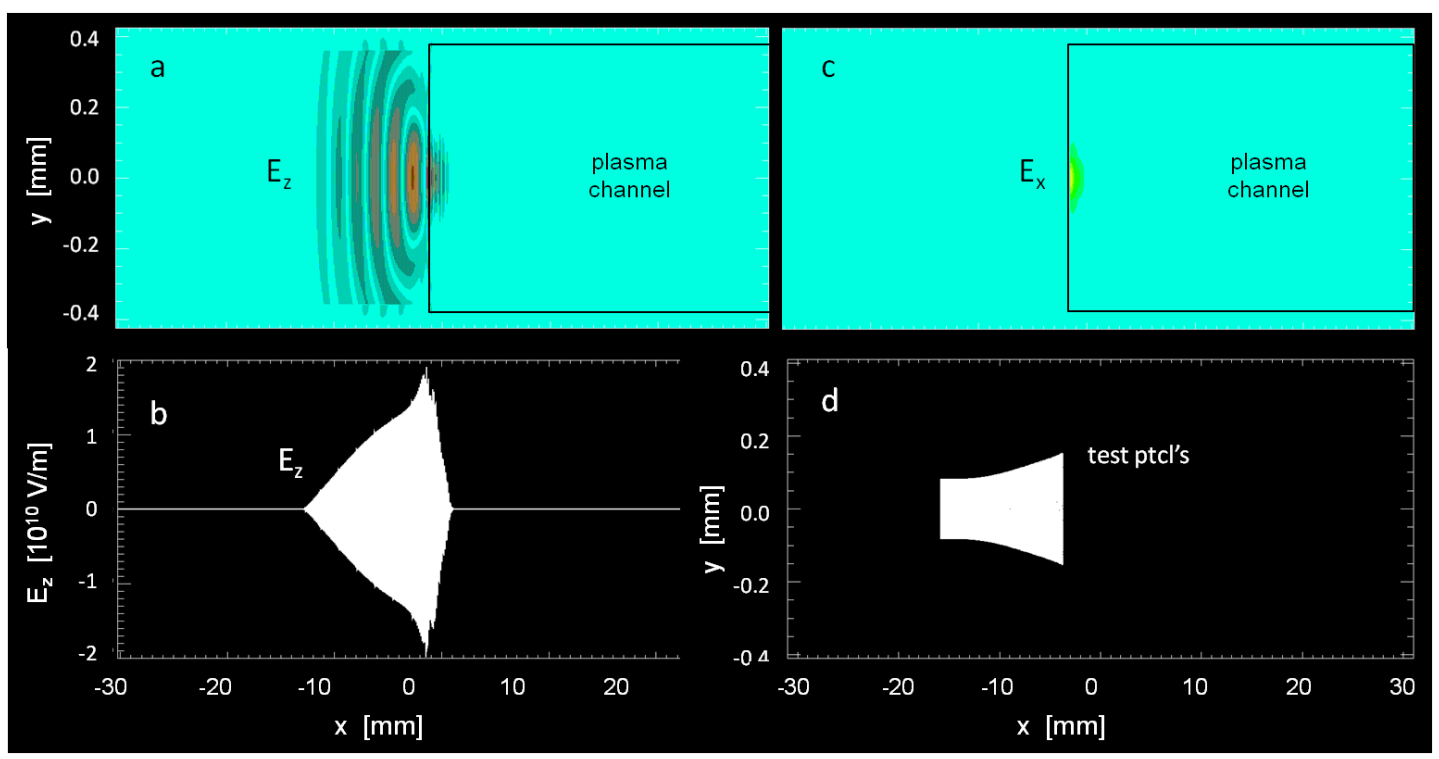

FIGURE 1. Example of a 2D boosted frame LWFA simulation is shown at early times, with laser pulse moving to the right and plasma channel moving to the left - a) contour plot of the laser electric field as it focuses to a waist at the leading edge of the channel; b) lineout of the laser electric field; c) contour plot of the longitudinal electric field at the left edge of the plasma; d) $x-y$ scatter plot of cold, $10 \mathrm{MeV}$ test-particles overlapping the laser pulse.

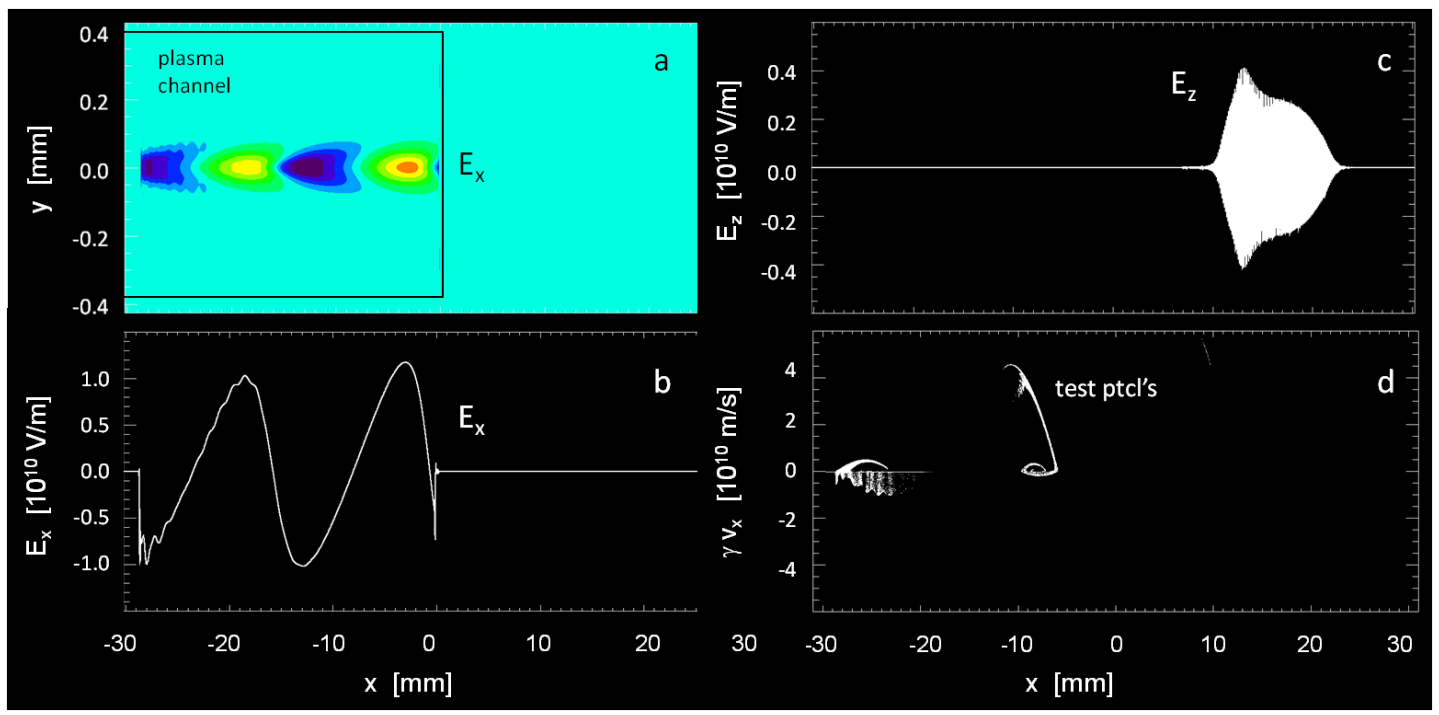

FIGURE 2. Same 2D boosted frame LWFA simulation from Fig. 1, later in time - a) contour plot of the longitudinal electric field in the plasma; b) lineout of the plasma wakefield; c) lineout of the laser electric field; d) longitudinal phase space scatter plot of accelerated test-particles behind the pulse.

For the simulation described by Fig.'s 1 and $2, \gamma_{b}=70$, yielding a theoretical speedup factor of $2 \gamma_{b}^{2}=9,800$. Due to various inefficiencies, which can be corrected in the future, the achieved speedup was $\sim 2,000 \mathrm{x}$ as compared to lab frame PIC simulation. 
Fig. 1 shows the laser pulse as it enters the plasma, the initial plasma wakefield, and a distribution of test-particles used to sample the wakefields and predict the maximum possible energy for an externally injected electron bunch. The longitudinal profile of the laser pulse is asymmetric in the boosted frame. In order to generate the laser pulse accurately, with no increase in the transverse size of the simulation domain, it is emitted from three walls, using the total field/scattered field technique [56].

Fig. 2 shows the plasma wakefield near the end of the simulation, the departing laser pulse, and the longitudinal phase space of the test-particles, some of which have been accelerated to high-energy and are following behind the laser pulse. The peak normalized momentum in the boosted frame, as seen in Fig. 2 d, corresponds to $\gamma \approx 157$. The corresponding value in the lab frame is $\gamma_{1 a b} \approx 2 \gamma_{b} \gamma \approx 2.2 \times 10^{4}$, corresponding to a peak electron energy of $11 \mathrm{GeV}$. This agrees well with the predictions of Ref. [43].

\section{HIGH-BRIGHTNESS PHOTOCATHODE ELECTRON SOURCES}

Photocathode electron sources can be "warm" or superconducting, rf or DC, a single (half) cell or multiple cells, and can employ any of a wide variety of photocathode technologies. The basic terminology is as varied as the technologies employed, but we use here the fairly generic term "electron gun". It is beyond the scope of this paper to discuss the various types of electron guns, but an excellent collection of reports summarizing international R\&D efforts on high-brightness photocathode electron sources has been recently collected [1].

Many high-brightness electron gun development efforts are motivated by "conventional" high-intensity linear accelerators for colliders, free-electron lasers (FEL), energy recovery linacs (ERL) or other applications that are not directly relevant to advanced concepts. However, all such efforts are expected to benefit AAC efforts in the future, and so the topic is integral to the AAC workshops. In particular, electron gun work at Argonne National Lab [2] and UCLA [3] is directly relevant to AAC.

There are two fundamental approaches to simulating electron guns - electrostatic and electromagnetic. Many codes have been developed for both approaches. Due to space limitations, only a few codes are discussed here.

\section{Electrostatic Gun Simulations}

The oldest and still most widely used approach to simulating electron guns is called "electrostatic," because the electron bunch is Lorentz transformed to its beam frame, where the Poisson equation is solved via electrostatic PIC for the electric fields, which are then transformed back into the lab frame in order to apply the corresponding force to the electron macro-particles. The oldest and most widely used code for this approach is PARMELA [4], which has been tested extensively (see e.g. [1,5]) and is generally used to benchmark new codes.

The more recently developed IMPACT-T code [6], uses the same general approach as PARMELA, but the Poisson solver is fully $3 \mathrm{D}$ and, like the particle dynamics, is parallelized to run efficiently on clusters and supercomputers. Also, IMPACT-T can "slice" the electron bunch longitudinally and calculate the corresponding fields independently (important near the photocathode, where large energy spread can 
otherwise prevent the sensible definition of a beam frame), then sum them up before applying the force to the electron macro-particles. Recent developments include the addition of a model for backstreaming ions [7], which can appear in high-duty factor electron guns via impact ionization of the background and then can be accelerated into the cathode.

\section{Electromagnetic Gun Simulations}

Electromagnetic PIC simulations of electron guns can in principle capture more physics than the electrostatic treatment. For high gradients and correspondingly rapid acceleration near the cathode, the electrostatic field calculation is not accurate. Likewise, beam loading of the rf fields (especially important near the cathode) cannot be modeled from first principles in the electrostatic approach. For high-charge, highduty-factor, high-Q electron guns, the high-order mode wakefields generated by each electron bunch could linger sufficiently long to accumulate within the resonant cavity and eventually perturb the particle dynamics of subsequent bunches - an effect that can only be modeled with fully electromagnetic PIC.

On the other hand, there are concerns with the electromagnetic approach. While an electrostatic Poisson solve need only grid up the spatial region around the electron bunch, an electromagnetic simulation must grid up the much larger domain occupying the full interior of the rf cavity. Also, the Courant stability condition on the time step for electromagnetic simulation means that many more steps must be simulated. As a result, electromagnetic simulations generally require orders of magnitude more computer time. Even so, there is concern that one might not sufficiently resolve the region near the photocathode, where nonlinear self-fields are dramatically affecting the subsequent beam dynamics. Also, the particle noise in an electromagnetic simulation could in some cases increase the emittance of the accelerated electron bunch, or drive artificially strong high-order modes. As a result, caution is required.

VORPAL [8] simulations of rf cavities have been shown to accurately capture several resonant modes in complicated structures $[9,10]$. Recently, VORPAL has been used to simulate [11] the half-cell SRF gun designed at Brookhaven National Lab [12], showing good agreement with PARMELA in a regime where both codes should be valid.

The Advanced Computation department at SLAC has developed the unique Pic3P electromagnetic PIC code [13,14], which uses the finite element (FE) technique to implement time-domain PIC, rather than the finite-difference time-domain (FDTD) method used by VORPAL and also by the commercial PIC codes MAFIA and MAGIC. The Advanced Computation department has also developed software, called TEM3P for modeling thermal effects $[15,16]$ on rf cavities. Pic3P has been shown to agree well with PARMELA for a case where both are valid (low current) and to agree with MAFIA for higher-currents (in disagreement with PARMELA), and to run much faster than MAFIA.

A completely different approach to the problem of electromagnetic gun simulations is to use Green's functions $[17,18]$. This approach is still in the early stages, requiring simple geometry and short electron bunches to be practical, but shows promise for the future. 


\section{Simulating Photocathode Physics and Diamond Amplification}

Improved and generalized photoemission models have been developed to include the effects of thermal and field emission $[19,20]$ and some simulation codes have begun to incorporate them [21]. A different type of electron/material interaction is key to the concept of diamond amplification of the electron charge in an rf electron gun [22], which is now being simulated with VORPAL [23].

\section{SIMULATION OF OTHER ADVANCED CONCEPTS}

It is beyond the scope of this paper to discuss simulation techniques for all advanced acceleration concepts; however, we briefly mention two more of them here. Explicit PIC can be used to simulate the acceleration of ions in laser-solid interactions - for example the VPIC code [24,25], which is heavily optimized for use on opteron clusters and in particular on the hybrid opteron/cell architecture of the RoadRunner supercomputer at LANL. This effective use of massively parallel computing power enables the use of huge meshes and large numbers of particles per cell.

Dielectric structures show great promise for producing high gradients in laserdriven cavities and novel light sources $[26,27,28]$. Because such structures often rely on a high-order mode (i.e. smaller wavelength than the fundamental), they can be electrically very large (many wavelengths across). This disparity of spatial scales can make the simulations an order of magnitude larger and slower than the comparable simulations for a metal $\mathrm{rf}$ cavity. Developing electromagnetic algorithms that are both stable and $2^{\text {nd }}$-order accurate in the presence of arbitrary dielectrics is still an active area of research [29].

\section{ACKNOWLEDGMENTS}

The authors received useful input from many members of the AAC community, and we acknowledge the on-going efforts and contributions of the VORPAL development team. This work was supported by the U.S. Department of Energy Office of Science, Office of High Energy Physics, under Contract Nos. DE-AC02-05CH11231, DEFG02-07ER84834 and DE-FC02-07ER41499 and by Tech-X Corporation. The computational resources of NERSC were used.

\section{REFERENCES}

1. "ICFA Beam Dynamics Newsletter," No. 46, Eds. M.A. Furman and W. Chou (August, 2008); http://icfa-usa.jlab.org/archive/newsletter/icfa_bd_nl_46.pdf

2. Ibid., J.W. Lewellen and J.G. Power, "Electron Gun Development at Argonne National Laboratory," Sec. 3.5, p. 73.

3. Ibid., J. Rosenzweig, "The High Brightness Electron Beam Physics and Photoinjector Technology Program at UCLA," Sec. 3.8, p. 119.

4. L. Young and J. Billen, "The Particle Tracking Code PARMELA," Proc. Part. Accel. Conf., 3521 (2003); PARMELA web site, http://laacg1.lanl.gov/laacg/services/serv_codes.phtml\#parmela

5. C. Limborg et al., "Comparison of PARMELA Simulations with Longitudinal Emittance Measurements at the SLAC Gun Test Facility," Proc. Part. Accel. Conf., 3216 (2003). 
6. J. Qiang, S. Lidia, R.D. Ryne and C. Limborg-Deprey, "Three-dimensional quasistatic model for high brightness beam dynamics simulation," Phys. Rev. ST/AB 9, 044204 (2006).

7. J. Qiang (private communication).

8. C. Nieter and J.R. Cary, "VORPAL: a versatile plasma simulation code," J. Comput. Phys. 196, 538 (2006).

9. J.R. Cary (private communication).

10 G.R. Werner and J.R. Cary, "Extracting degenerate modes and frequencies from time-domain simulations with filter-diagonalization," J. Comput. Phys. 227, 5200 (2008).

11 K. Paul, D.A. Dimitrov, R. Busby, D.L. Bruhwiler, D. Smithe, J.R. Cary, J. Kewisch, D. Kayran, R. Calaga and I. Ben-Zvi, "Half-Cell RF Gun Simulations with the Electromagnetic Particle-in-Cell Code VORPAL," in these proceedings, 2008.

12 I. Ben-Zvi and I. Bazarov, Nucl. Instr. and Meth. in Phys. Res. A 557, 337 (2006).

13 A. Candel, A. Kabel, L. Lee, Z. Li, C. Limborg, C. Ng, E. Prudencio, G. Schussman, R. Uplenchwar and K. Ko, "Parallel Finite Element Particle-in-Cell Code for Simulations of Space-Charge Dominated Beam-Cavity," Proc. Part. Accel. Conf., 3216 (2007).

14 A. Candel et al., in these proceedings, 2008.

15 V. Akcelik, A. Candel, A. Kabel, L-Q. Lee, Z. Li, C-K. Ng, L. Xiao and K. Ko, "Parallel Computation of Integrated Electromagnetic, Thermal and Structural Effects for Accelerator Cavities," Proc. European Part. Accel. Conf., 1724 (2008).

16 C. Ng, V. Akcelik, A. Candel et al., "Design and optimization of large accelerator systems through high-fidelity electromagnetic simulations," J. Physics: Conf. Series 125, 012003 (2008).

17 M. Hess and C.S. Park, "A Multislice Approach for Electromagnetic Green's Function Based Beam Simulations," Proc. Part. Accel. Conf., 3531 (2007).

18 M. Hess et al., in these proceedings, 2008.

19 K.L. Jensen, B.L. Jensen, E.J. Montgomery, D.W. Feldman, P.G. O'Shea, N. Moody, "Theory of photoemission from cesium antimonide using an alpha-semiconductor model," J. Applied Phys. 104, 044907 (2008).

20 K.L. Jensen, "General formulation of thermal, field, and photoinduced electron emission," $J$. Applied Phys. 102, 024911 (2007).

21 D.A. Dimitrov, D.L. Bruhwiler, J.R. Cary, P. Messmer, P. Stoltz, K.L. Jensen, D.W. Feldman and P.G. O'Shea, "Development of Advanced Models for 3D Photocathode PIC," Proc. Part. Accel. Conf., 2583 (2005).

22 J. Smedley, I. Ben-Zvi, A. Burrill, X. Chang, J. Grimes, T. Rao, Z. Segalov and Q. Wu, "Electron Amplification in Diamond," in $12^{\text {th }}$ Advanced Accelerator Concepts Workshop, edited by M. Conde and C. Eyberger, AIP Conference Proceedings 877, American Institute of Physics, Melville, NY, 2006, p. 672.

23 D.A. Dimitrov, R. Busby, D.L. Bruhwiler, J.R. Cary, I. Ben-Zvi, T. Rao, X. Chang, J. Smedley and Q. Wu, "3D Simulations of Secondary Electron Generation and Transport in a Diamond Amplifier for Photocathodes," Proc. Part. Accel. Conf., 3555 (2007).

24 K.J. Bowers, B.J. Albright, L. Yin, B. Bergen and T.J.T. Kwan, "Ultrahigh performance three dimensional electromagnetic relativistic kinetic plasma simulation," Phys Plasmas 15, 055703 (2008).

25 B.J. Albright et al., in these proceedings, 2008.

26 B. Cowan, "Three-dimensional dielectric photonic crystal structures for laser-driven acceleration," Phys. Rev. ST/AB 11, 011301 (2008); B. Cowan, in these proceedings, 2008.

27 T. Plettner and R. L. Byer, "Proposed dielectric-based microstructure laser-driven undulator," Phys. Rev. ST/AB 11, 030704 (2008).

28 C.M.S. Sears, E.R. Colby, B. Cowan, R. Ischebeck, C.M. McGuinness, R. Noble, R.H. Siemann, J.E. Spencer, D. Walz, R.L. Byer and T. Plettner, "Optical Wakefield from a Photonic Bandgap Fiber Accelerator," Proc. Part. Accel. Conf., 3106 (2007).

29 G.R. Werner and J.R. Cary, "A stable FDTD algorithm for non-diagonal, anisotropic dielectrics," $J$. Comput. Phys. 226, 1085 (2008).

30 W.P. Leemans, B. Nagler, A.J. Gonsalves, C. Toth, K. Nakamura, C.G.R. Geddes, E. Esarey, C.B. Schroeder and S.M. Hooker, "GeV electron beams from a centimetre-scale accelerator," Nature Physics 2, 696 (2006). 
31 E. Esarey, P. Sprangle, J. Krall and A. Ting, "Overview of plasma-based accelerator concepts," IEEE Trans. Plasma Science 24, 252 (1996).

32 R.A. Hemker et al., Lecture Notes in Computational Science 2331, 342 (2002).

33 S.P.D. Mangles, C.D. Murphy, Z. Najmudin, A.G.R. Thomas, J.L. Collier, A.E. Dangor, E.J. Divall, P.S. Foster, J.G. Gallacher, C.J. Hooker et al., "Monoenergetic beams of relativistic electrons from intense laser-plasma interactions," Nature 431, 535 (2004).

34 F.S. Tsung, W. Lu, M. Tzoufras, W.B. Mori, C. Joshi, J.M. Vieira, L.O. Silva, and R. A. Fonseca, "Simulation of monoenergetic electron generation via laser wakefield accelerators for 5-25TW lasers," Phys. Plasmas 13, 056708 (2006).

35 J.M. Vieira et al., IEEE Transactions on Plasma Science 36, 1722 (2008).

36 C.G.R. Geddes, C. Toth, J. van Tilborg, E. Esarey, C.B. Schroeder, D.L. Bruhwiler, C. Nieter, J.R. Cary, and W. Leemans, "High-quality electron beams from a laser wakefield accelerator using plasma-channel guiding," Nature 431, 538 (2004).

37 J.R. Cary, R. Giacone, C. Nieter and D.L. Bruhwiler, "Clean beams from laser wake-field accelerators via optical injection with a cleanup pulse," Phys. Plasmas 12, 056704 (2005).

38 C.G.R. Geddes et al., Phys. Rev. Lett. 100, 215004 (2008).

39 K. Paul, D.L. Bruhwiler, B. Cowan, J.R. Cary, C. Huang, F.S. Tsung, W.B. Mori, E. CormierMichel, C.G.R. Geddes, E. Esarey, S. Martins, R.A. Fonseca and L.O. Silva, "Benchmarking the codes VORPAL, OSIRIS and QuickPIC with Laser Wakefield Acceleration Simulations," in this proceedings (2008).

40 C.K. Birdsall and A.B. Langdon, Plasma Physics via Computer Simulation, New York: McGrawHill, 1985.

41 E. Cormier-Michel, B.A. Shadwick, C.G.R. Geddes, E. Esarey, C.B. Schroeder and W.P. Leemans, "Unphysical kinetic effects in particle-in-cell modeling of laser wakefield accelerators," Phys. Rev. E 78, 016404 (2008).

42 C.G.R. Geddes et al., in this proceedings.

43 E. Cormier-Michel, C.G.R. Geddes, E. Esarey, C.B. Schroeder, D.L. Bruhwiler, K. Paul, B. Cowan and W.P. Leemans, "Scaled simulations of a $10 \mathrm{GeV}$ accelerator," in this proceedings.

44 D. Gordon, W. Mori and T. Antonsen, Jr., IEEE Trans. Plasma Science 28, 1135 (2000).

45 P. Messmer and D.L. Bruhwiler, Phys. Rev. ST Accel. Beams 9, 031302 (2006).

46 B. Cowan, D.L. Bruhwiler, P. Messmer, K. Paul, C.G.R. Geddes, E. Esarey and E. Cormier-Michel, "Laser wakefield simulation using a speed-of-light frame envelope model," in this proceedings (2008).

47 C.K. Huang et al., J. Comput. Phys. 217, 658 (2006).

$48 \mathrm{~W}$. Mori (private communication).

49 J.-L. Vay, "Noninvariance of space- and time-scale ranges under a Lorentz transformation and the implications for the study of relativistic interactions," Phys. Rev. Lett. 98, 130405 (2007).

50 J.-L. Vay, "Simulation of beams or plasmas crossing at relativistic velocity," Phys. Plasmas 15, 056701 (2008).

51 C.G.R. Geddes, D.L. Bruhwiler, J.R. Cary, W.B. Mori, J-L. Vay, S.F. Martins, T. Katsouleas, E. Cormier-Michel, W.M. Fawley, C. Huang, X. Wang, B. Cowan, V.K. Decyk, E. Esarey, R.A. Fonseca, W. Lu, P. Messmer, P. Mullowney, K. Nakamura, K. Paul, G.R. Plateau, C.B. Schroeder, L.O. Silva, Cs. Toth, F.S. Tsung, M. Tzoufras, T. Antonsen, J. Vieira and W.P. Leemans, "Computational studies and optimization of wakefield accelerators," J. Physics: Conf. Series 125, 012002 (2008).

52 S. Martins et al., in this proceedings (2008).

53 W.M. Fawley et al., in this proceedings (2008).

54 W. Lu, M. Tzoufras, F.S. Tsung, C. Joshi, W.B. Mori, J. Vieira, R.A. Fonseca and L.O. Silva, "Designing LWFA in the Blowout Regime," Proc. Part. Accel. Conf., 3050 (2007).

55 M. Tzoufras, W. Lu, F.S. Tsung, C. Huang, W.B. Mori et al., "The Physical Picture of Beam Loading in the Blowout Regime," Proc. Part. Accel. Conf., 3061 (2007).

56 Taflove \& Hagness, Computational Electrodynamics: The Finite-Difference Time-Domain Method, 2nd ed., Norwood, MA: Artech House, 2000.

57 W. Lu, M. Tzoufras C. Joshi et al., "Generating multi-GeV electron bunches using single stage laser wakefield acceleration in a 3D nonlinear regime," Phys. Rev. ST/AB 10, 061301 (2007). 